INRA Prod. Anim., 2004, 17 (3), 167-175

\section{A. NEVEU}

UMR EQHC, INRA - ENSAR, 65 Rue de Saint-Brieuc, CS 84215, F-35042 Rennes Cedex

Courriel :neveu@rhoazon.inra.fr

\title{
La raniculture est-elle une alternative à la récolte ? Etat actuel en France
}

Les grenouilles comestibles (environ 50 espèces) ne représentent qu'une très faible partie de la diversité des Amphibiens (4000 espèces), et même des Ranidae (700 espèces) dont les représentants modernes n'ont guère évolué depuis le Trias (-220 millions d'années). Beaucoup d'espèces sont trop petites (quelques grammes) et souvent trop toxiques (Dendrobates) pour entrer dans la consommation humaine, sauf sous forme de drogue (Bufo marinus). Il existe du reste une longue histoire de l'utilisation des grenouilles en médecine traditionnelle ou pour empoisonner les flèches, sans parler de leur présence dans de nombreux folklores (Wasserman 1990).

\section{Résumé}

La grenouille est apparue dans la gastronomie européenne au cours du 16ème siècle. La plupart des grenouilles consommées sont prélevées sur des stocks naturels, mais ceux-ci ont fortement diminué au cours du $20^{\text {ème }}$ siècle du fait de l'augmentation de l'activité humaine (augmentation des prélèvements, diminution des habitats favorables). La France est le principal pays importateur avec 3000-4000 t/an de cuisses surgelées en provenance des pays d'Extrême-Orient et 700-800 t/an de grenouilles vivantes. Au cours des dernières années, des essais d'élevage intensif de grenouilles ont été conduits dans plusieurs pays. Des essais ont été mis en place dans les années 80 dans l'ouest de la France pour tester les capacités d'élevage de grenouilles du complexe esculenta (deux espèces parentales : Rana lessonae et $R$. ridibunda et un hybride : $R$. esculenta). $R$. ridibunda s'est avérée la plus intéressante pour l'élevage intensif et a fait l'objet d'un essai de longue durée. Au début de l'essai, les femelles effectuaient plusieurs pontes par an avec une fertilité satisfaisante. Après une dizaine d'années d'élevage, la fécondité et la fertilité ont diminué, sans que les causes de ces diminutions soient totalement élucidées. Le développement des têtards dure de 2 à 3 mois.Les grenouillettes peuvent être alimentées avec des granulés dès la métamorphose. Après 40 jours, le taux de survie est de $92 \%$ et la taille moyenne de $32 \mathrm{~mm}$. La durée d'élevage depuis le sevrage est de 10 à 12 mois, avec une production de 20 à $30 \mathrm{~kg} / \mathrm{m}^{2}$ pour une température comprise entre 15 et $25^{\circ} \mathrm{C}$. Une alternative à la production intensive est de reconstituer les stocks naturels. Depuis 1986, plusieurs essais ont été conduits avec $R$. temporaria, qui associent l'élevage intensif des têtards en étang, le lâcher des grenouillettes dans la nature et le suivi du retour des adultes qui reviennent pondre sur leur lieu de naissance après deux ou trois ans (taux de retour d'environ $6 \%$ ). Le choix entre ces deux voies doit également tenir compte des contraintes réglementaires et socio-économiques.
Récemment l'analyse de leur peau a révélé une richesse en divers composants peptidiques d'intérêt (Amiche et al 1996).

Plusieurs pays européens ont progressivement intégré la grenouille dans leur gastronomie à partir du 16ème siècle. Plus récemment, le développement de la chaîne du froid a permis une forte augmentation de sa consommation qui est aujourd'hui de 8000 à 10000 tonnes de cuisses par an dans l'ensemble des pays concernés (Hardouin 1994). La plupart des grenouilles consommées sont prélevées sur des stocks naturels. Mais ces stocks sont en forte diminution, particulièrement depuis les années 80 (Collins et Storfer 2003), sous l'influence conjuguée de la régression des habitats favorables et de l'augmentation des activités humaines, en particulier de la récolte (Green 1997).

\section{1 / Importance économique en France}

En France, les grenouilles sont consommées traditionnellement dans les zones de marais (Vendée, Dombes ...), mais aussi dans certaines zones de moyenne altitude (Ardennes, Jura ...). Dans le premier cas, il s'agit de grenouilles vertes, ou Rana esculen$t a$, dans le second de grenouille rousse ou Rana temporaria.

Jusque vers les années 60, les récoltes régionales, de 40 à 70 t/an (grenouilles entières) étaient suffisantes pour la consommation nationale. Par la suite, la réduction des stocks et les progrès en matière de conservation ont conduit à importer des animaux vivants puis des cuisses surgelées et, finalement, à s'interroger sur les possibilités d'élevage. En effet, depuis 1980, les prélèvements commerciaux dans la nature sont interdits en France et seule la pêche de loisir reste tolérée sur $R$. esculenta et $R$. temporaria selon des modalités proches de celles appliquées au poisson. 
Les importations se sont développées selon deux filières (tableau 1) : cuisses surgelées destinées à la grande distribution, à partir des pays d'Extrême Orient (Indonésie, Chine ...) et animaux vivants destinés surtout à la restauration, à partir de pays péri-méditerranéens (Turquie, Egypte, Albanie ...). Cette filière est en régression avec des prix en augmentation. Les cuisses surgelées proviennent d'individus sauvages pour l'Indonésie, voire d'individus issus de repeuplements, mais de plus en plus d'individus d'élevage pour la Chine, alors que les importations de grenouilles vivantes proviennent d'individus sauvages.

Pour un animal pesant entre 80 et $100 \mathrm{~g}$, le rendement de découpe est de 60 à $80 \%$ de carcasse, soit 33 à $47 \%$ en cuisses avec une découpe courte («parisienne») et 38 à $51 \%$ avec une découpe longue («lyonnaise»). Le poids des ovaires peut représenter de 20 à $25 \%$ du poids total. La viande de grenouille est peu persillée, les lipides étant essentiellement localisés autour des viscères.

\section{2 / Le problème du choix de l'espèce pour l'élevage}

\section{1 / Historique}

Les premiers essais d'élevage ont probablement été ceux de Viosca entre 1915 et 1917 avec la grenouille taureau ( $R$. catesbeiana), espèce séduisante par la taille de ses cuisses : de 300 à $400 \mathrm{~g}$ pour des animaux de $1 \mathrm{~kg}$ (Viosca 1934). Cet élevage est longtemps resté extensif, avec un simple contrôle de la prédation dans des étangs aménagés. Ce n'est qu'au cours des années 80 que des essais d'élevage plus intensif ont été effectués, d'abord en Louisiane pour couvrir avant tout le marché de la grenouille vivante pour l'enseignement. Mais les coûts de main d'œuvre se sont vite avérés élevés. D'autres essais ont ensuite été conduits au Brésil et à Taïwan, avec de bons résultats sur aliments artificiels (Chen 1990, Lima et Agostino 1992). La grenouille taureau a également été introduite dans de nombreux pays pour diverses tentatives d'élevage : Italie, Grèce, Japon... Certains élevages perdurent en Chine et dans quelques pays sud-américains. Elle a été introduite récemment en France, dans le Bordelais à partir duquel elle se répand (Neveu 1997). En région chaude, cette grenouille a un développement assez lent (1 à 2 ans). Aussi, d'autres espèces au développement plus rapide ( $R$. crassa) donnent lieu à quelques élevages (Taïwan) $: R$. crassa peut produire 3 générations par an et les rendements s'élèvent à $20 \mathrm{~kg} / \mathrm{m}^{2}$ (Chen 1990). De même, $R$. rugulosa est en cours d'intensification en Thailande, elle peut atteindre $150 \mathrm{~g}$ en 5 mois (Somsueb et Boonyaratpalin 2001).

Les importations européennes de cuisses surgelées proviennent des espèces d'Extrême Orient : $R$. tigrina, $R$. crassa et $R$. catesbeia$n a$, alors que les importations d'animaux vivants concernent le groupe européen $R$. ridibunda, éclaté aujourd'hui en plusieurs espèces.

\section{2 / Quel choix pour la France?}

En France, les grenouilles (genre Rana) sont représentées par deux groupes d'animaux :

- les grenouilles vertes avec 2 complexes (c'est-à-dire l'association au moins d'une espèce parentale et d'un hybride) : le complexe esculenta avec $R$. lessonae, $R$. ridibunda comme espèces parentales et $R$. esculenta, hybride naturel, et le complexe grafi avec $R$. perezi comme espèce parentale et $R$. grafi comme hybride ;

- les grenouilles rousses avec $R$. temporaria, $R$. dalmatina, $R$. iberica et $R$. arvalis.

Le premier groupe est constitué d'animaux aux moeurs aquatiques, tant au stade larvaire qu'adulte, et qui peuplent plutôt les plans d'eau assez grands et permanents. Le deuxième groupe est constitué d'animaux terrestres au stade adulte ; seule la reproduction et le développement des têtards s'effectuent dans les milieux aquatiques souvent de faible taille voire temporaires. Aussi ces espèces, surtout $R$. temporaria, présentent un phénomène de homing, c'est-à-dire un retour des adultes sur leur lieu de naissance. Sorte de garantie pour l'espèce de retrouver de l'eau dans la mesure où les milieux aquatiques colonisés peuvent être aléatoires.

Tableau 1. Evolution des importations de grenouilles en France de 1965 à 2002 (tonnages annuels) et des prix selon les catégories et les époques (source: Statistiques des Douanes françaises).

\begin{tabular}{|c|c|c|c|c|}
\hline & \multicolumn{2}{|c|}{ Cuisses surgelées } & \multicolumn{2}{|c|}{ Grenouilles vivantes } \\
\hline Année & $\begin{array}{c}\text { Tonnage } \\
\text { (t/an) }\end{array}$ & $\begin{array}{c}\text { Prix (€/kg) } \\
\text { (Asie) }\end{array}$ & $\begin{array}{c}\text { Tonnage } \\
\text { (t/an) }\end{array}$ & $\begin{array}{c}\text { Prix (€/kg) } \\
\text { (Turquie, Egypte) }\end{array}$ \\
\hline 1965 & 369 & & 453 & \\
\hline 1970 & 713 & \multirow{2}{*}{$2,3-2,6$} & 1084 & \multirow{2}{*}{$1,2-1,4$} \\
\hline 1975 & 1900 & & 895 & \\
\hline 1980 & 4046 & & 746 & \\
\hline 1985 & 2950 & & 710 & \\
\hline 1990 & 2578 & \multirow{2}{*}{$3,6-3,9$} & 904 & \multirow{2}{*}{$3,0-3,8$} \\
\hline 1995 & 4250 & & - & \\
\hline 2000 & 3150 & \multirow{2}{*}{$\begin{array}{l}\text { 2,1-2,6 (élevage Chine) } \\
\text { 3,8-4,5 (sauvage Indonésie) }\end{array}$} & 553 & \multirow{2}{*}{$5,5-6,0$} \\
\hline 2002 & 3290 & & 326 & \\
\hline
\end{tabular}


Traditionnellement toutes ces espèces sont consommées (ou peuvent l'être), mais les principales espèces exploitées sont les grenouilles vertes (lato sensu) et $R$. temporaria pour les grenouilles rousses.

La réglementation française interdit toute introduction d'espèce nouvelle, aussi les expérimentations effectuées par l'INRA dès les années 80 ont-elles porté sur le potentiel des espèces locales. Les essais d'intensification sur $R$. temporaria, espèce terrestre à l'état adulte, ont été décevants (sauf au niveau des têtards), en relation avec la fragilité des grenouillettes de cette espèce d'espaces frais et ombragés (bois, forêts, prairies), dont l'aire de répartition remonte jusqu'au nord de la Scandinavie et qui présente une très grande plasticité dans son cycle de vie selon la latitude et l'altitude (Miaud et Guyétant 1998).

Les premiers essais sur le complexe des grenouilles vertes (esculenta), à mours aquatiques, se sont révélés rapidement plus prometteurs avec, successivement, des essais d'élevage en parcs aménagés, puis d'élevage plus intensif avec une alimentation à base de proies vivantes et, depuis les années 90, d'élevage avec une alimentation granulée. Mais ce groupe d'espèces présente une particularité génétique : un hybride naturel ( $R$. esculenta), normalement stérile en auto-croisement, mais fertile en rétro-croisement avec l'une ou l'autre des espèces parentales $(R$. lessonae, $R$. ridibunda), qui est très fréquent dans les populations naturelles.

Autrement dit, trois cas peuvent se présenter, en particulier pour le nord et l'ouest de la France :

- soit un croisement entre espèces parentales, $R$. lessonae (LL) ou $R$. ridibunda (RR) (couples LL x LL, RR x RR), dans ce cas la fertilité est normale et la F1 est constituée d'individus semblables aux parents (LL ou $\mathrm{RR}$ ). Il peut y avoir quelques croisements entre les deux espèces (couples LL x RR), la fertilité est alors normale et la F1 est constituée d'hybrides primaires (RL) ;

- soit un croisement entre une espèce parentale et l'hybride $R$. esculenta (RL) (couples RL x LL, plus rarement RL x RR). Dans ce cas, la fertilité est normale (les mâles RL sont cependant peu fertiles, contrairement aux femelles). La F1 est constituée d'hybrides RL. Il y a une élimination pré-méiotique d'un des génomes (le plus souvent L, les ovules sont donc R). Il s'agit alors d'une reproduction de type hybridogénétique avec transmission clonale des caractères. Le croisement avec ridibunda (RL x RR) peut donner soit $R R$ soit $R L$ selon la zone géographique ;

- soit un auto-croisement de l'hybride (RL x $\mathrm{RL}$ ) normalement infertile, mais qui quelquefois donne des ridibunda (RR). A noter que cette infertilité se traduit soit par des mortalités des embryons, soit des têtards même bien développés.

Il peut par ailleurs y avoir un flux de triploïdes, d'intensité variable selon les sites. Les femelles hybrides produisent souvent des ovules haploïdes et diploïdes (RL, RR, LL) qui donnent en F1 des individus triploïdes (RLL, RRL). Ainsi dans l'ouest de la France, les mâles triploïdes RLL sont régulièrement présents ; ils peuvent atteindre 90 à $95 \%$ des effectifs dans certaines populations. Ils peuvent être détectés, en dehors de leur morphologie externe proche de $R$. lessonae, par une analyse biométrique des érythrocytes (surface des érythrocytes : 200 à $350 \mu^{2}$ pour $2 \mathrm{n}$, 350 à $520 \mu^{2}$ pour $3 n$ ). Leur intérêt réside dans le fait qu'ils peuvent rendre le croisement entre hybrides (RL x RLL) normalement fertile (pour des raisons mal connues) avec formation de RR et RLL.

Cette structuration des peuplements concerne le nord de la France (au-delà d'une ligne La Rochelle-Lyon-Genève) alors qu'au sud existe un autre complexe avec $R$. perezi (PP) (seule espèce en Espagne) et son hybride hybridogénétique $R$. grafi (RP) (issu d'un croisement originel avec $R$. ribidunda).

Les capacités d'élevage de divers taxons ont été testées à partir de géniteurs capturés dans l'ouest de la France, dans une zone comprise entre les villes de Rennes et Redon (Pays de Vilaine). Rapidement, l'espèce parentale ridibunda s'est révélée plus facile à domestiquer que les génotypes LL, RL et RLL. Cependant, depuis l'année 2000, grâce au progrès de la technique d'élevage, ces derniers sont aussi élevés en milieu artificiel, mais avec une survie moindre et une croissance plus réduite, ce qui les rend inaptes à un élevage de production. Ainsi, leur poids à un an est de 7 à 9 g., avec néanmoins une maturité sexuelle apparemment normale (surtout pour les lessonae), contre 30 à $50 \mathrm{~g}$ pour les ridibunda de même âge et de même origine (F1).

\section{3 / L'élevage intensif de R. ridibunda}

Les géniteurs fondateurs ont été capturés près de la ville de Rennes. Le nombre initial de pontes viables a été de 7 ( $=7$ femelles fondatrices). Comme dans de nombreuses régions, il est difficile de connaître l'histoire de ces ridibunda, espèce par ailleurs totalement protégée par la réglementation. Ce qui a priori peut paraître paradoxal dans la mesure où actuellement il est difficile de déterminer l'origine de l'espèce. Il semblerait qu'initialement elle n'ait été présente que dans l'est de la France, mais elle peut aussi être auto-produite dans certaines populations d'hybrides ; l'importance de ce phénomène est mal connue. Les importations régulières de ce type d'animaux (d'origine méditerranéenne) pour la consommation humaine et pour l'enseignement ont été à l'origine de nombreuses fuites de stockage, auxquelles s'ajoutent des introductions volontaires dans certains essais d'élevage extensif. Cela peut expliquer la répartition en taches de cette espèce à travers pratiquement toute la France à ce jour.

R. ridibunda a un cycle de développement semblable à celui des autres grenouilles vertes. Dans la nature, la ponte a lieu dans les eaux stagnantes à partir de fin mars jusqu'à fin juin, le développement des têtards dure 4 à 
5 mois, une partie de la population peut hiverner pour une métamorphose en mai/juin l'année suivante. La maturité sexuelle est atteinte en 2-3 ans dans les conditions normales.

Le contrôle thermique partiel permet de raccourcir ce cycle, de même que l'alimentation artificielle accélère la croissance.

\section{1 / La reproduction}

La reproduction peut s'effectuer en groupe ou par couple isolé, par exemple dans des cages nuptiales de $0,5 \mathrm{~m}^{2}$ avec environ $10 \mathrm{~cm}$ d'eau.

Le maximum d'activité sexuelle est relevé en avril, avec $52 \%$ des pontes (sous serre froide). Il est possible d'obtenir des pontes dès février en réchauffant progressivement les animaux hibernant, les pontes s'effectuent alors environ 3 à 4 semaines après le début du réchauffement. Les stimulations sont possibles avec des injections de LH-RH (20 $\mu \mathrm{g} / \mathrm{individu})$, mais leurs résultats sont variables si l'on ne connaît pas l'état de maturité des gonades. La variabilité individuelle est très forte en élevage, contrairement aux animaux sauvages plus synchrones.

\section{a / Fécondité}

$\mathrm{Au}$ début du processus d'élevage, certaines femelles pouvaient effectuer plusieurs pontes d'importance décroissante depuis mars jusqu'à juillet, les records ayant été de 5 pontes, observés chez deux femelles (tableau 2). Ce phénomène a presque disparu au cours des années suivantes où l'on observe la disparition de la $3^{\text {ème }}$ ponte et une forte réduction de la $2^{2 \text { ème }}$.

Le nombre d'œufs en $1^{\text {ère }}$ ponte dépend de la taille de la femelle :

$\mathrm{N}=0,0009 \mathrm{LT}^{3,33}(\mathrm{n}=39, \mathrm{r}=0,70)$, LT étant la longueur en mm entre la pointe du museau et l'urostyle. Plus simplement, $\mathrm{N}=25,5 \mathrm{P}, \mathrm{P}$ étant le poids en grammes.

Le nombre d'œufs par saison dépend de la capacité de la femelle à effectuer des pontes multiples. Ainsi pour un lot de 28 femelles, de 90 à $98 \mathrm{~mm}$, les femelles pondant une seule fois ont donné en moyenne 2215 œufs $(\zeta=1085, \mathrm{n}=9)$, celles pondant deux fois ont donné 3542 œufs ( 1 ère ponte : $2560 \pm 715$, $2^{\text {ème }}$ ponte : $982 \pm 394, \mathrm{n}=15$ ) et celles pondant trois fois une moyenne de 5658 œufs ( $1^{\text {èr }}$ ponte : $3034 \pm 1910,2^{\text {ème }}$ ponte $1476 \pm 366$, $3^{\text {ème }}$ ponte $\left.1148 \pm 206, \mathrm{n}=3\right)$. Enfin une femelle a donné 8650 en 4 pontes (4500 - 1400 1450 - 1300).

\section{b / Fertilité}

Au début de l'élevage, la fertilité à la 1ère ponte était inférieure à celle observée lors des pontes suivantes. Ceci semblait lié à la lenteur du développement des ovocytes au cours de l'hiver malgré une température assez élevée (serre) (tableau 3). En revanche,

Tableau 2. Evolution de la fécondité de la souche d'élevage de R. ridibunda au cours des années.

Evolution du pourcentage de femelles pondeuses présentant des pontes multiples au cours d'une même année ( $n c=$ nombres de couples contrôlés).

\begin{tabular}{|c|c|c|c|c|c|c|c|c|c|}
\hline Ponte & $\begin{array}{c}1986 \\
(\mathrm{nc}=4)\end{array}$ & $\begin{array}{c}1987 \\
(\mathrm{nc}=17)\end{array}$ & $\begin{array}{c}\mathbf{1 9 8 8} \\
(\mathrm{nc}=26)\end{array}$ & $\begin{array}{c}1989 \\
(\mathrm{nc}=23)\end{array}$ & $\begin{array}{c}1990 \\
(\mathrm{nc}=14)\end{array}$ & $\begin{array}{c}1991 \\
(\mathrm{nc}=16)\end{array}$ & $\begin{array}{c}1992 \\
(\mathrm{nc}=25)\end{array}$ & $\begin{array}{c}1993 \\
(\mathrm{nc}=24)\end{array}$ & $\begin{array}{c}1994 \\
(\mathrm{nc}=23)\end{array}$ \\
\hline $\mathrm{n}^{\circ} 2$ & 75 & 82 & 34 & 61 & 20 & 50 & 46 & 66 & 21 \\
\hline $\mathrm{n}^{\circ} 3$ & 75 & 70 & 23 & 5 & 7 & 18 & 7 & 16 & 0 \\
\hline $\mathrm{n}^{\circ} 4$ & 50 & 35 & 0 & 0 & 0 & 0 & 0 & 4 & 0 \\
\hline $\mathrm{n}^{\circ} 5$ & 25 & 6 & 0 & 0 & 0 & 0 & 0 & 0 & 0 \\
\hline
\end{tabular}

Evolution de la fécondité réelle des diverses pontes annuelles ( $n$ : nombre de pontes examinées, $m$ : nombre moyen d'œufs par ponte, $\zeta$ : écart type sur $m$ ).

\begin{tabular}{|c|c|c|c|c|c|c|}
\hline & \multicolumn{3}{|c|}{$\mathbf{1 9 8 6 - 1 9 8 7}$} & \multicolumn{3}{c|}{$\mathbf{1 9 9 2 - 1 9 9 3}$} \\
Ponte & $\mathbf{n}$ & $\mathbf{m}$ & $\boldsymbol{\zeta}$ & $\mathbf{n}$ & $\mathbf{m}$ & $\boldsymbol{\zeta}$ \\
\hline $\mathrm{n}^{\circ} 1$ & 22 & 2587 & 1326 & 49 & 2144 & 668 \\
\hline $\mathrm{n}^{\circ} 2$ & 17 & 1088 & 454 & 30 & 824 & 423 \\
\hline $\mathrm{n}^{\circ} 3$ & 14 & 945 & 369 & 6 & 501 & 190 \\
\hline $\mathrm{n}^{\circ} 4$ & 8 & 932 & 435 & 1 & 750 & 0 \\
\hline $\mathrm{n}^{\circ} 5$ & 2 & 870 & 240 & 1 & 460 & 0 \\
\hline
\end{tabular}

Tableau 3. Evolution au cours des années de la fertilité moyenne (pourcentage de têtards éclos) des diverses pontes annuelles de la souche d'élevage.

\begin{tabular}{|c|c|c|c|c|c|c|c|c|c|c|c|}
\hline Ponte & 1987 & 1988 & 1989 & 1990 & 1991 & 1992 & 1993 & 1994 & 2001 & 2002 & 2003 \\
\hline $\mathrm{n}^{\circ} 1$ & 37 & 80 & 48 & 48 & 85 & 62 & 35 & 57 & 12 & 27 & 35 \\
\hline $\mathrm{n}^{\circ} 2$ & 66 & 100 & 91 & 69 & 88 & 81 & 79 & 52 & $?$ & $?$ & $?$ \\
\hline $\mathrm{n}^{\circ} 3$ & 70 & 100 & 97 & 86 & 96 & 100 & 86 & - & - & - & - \\
\hline $\mathrm{n}^{\circ} 4$ & 50 & 100 & - & - & - & - & - & - & - & - & - \\
\hline $\mathrm{n}^{\circ} 5$ & 100 & - & - & - & - & - & - & - & - & - & - \\
\hline
\end{tabular}


lorsque les ovocytes se développaient rapidement, la fertilité était de 80 à $95 \%$, c'est-à-dire semblable à celle observée chez les animaux sauvages. Mais, au cours des dernières années d'élevage, la fertilité s'est réduite progressivement, phénomène que l'on retrouve aussi dans les élevages au Brésil ou en Chine, mais dont les causes exactes restent encore difficiles à cerner. Le ré-ensauvagement d'individus de l'élevage (fuites externes) permet un retour à une forte fertilité. De même, le rétro-croisement mâle sauvage $\mathrm{x}$ femelle d'élevage peut permettre de retrouver une fertilité normale, bien que celle-ci reste souvent très variable selon les couples.

Finalement des données en cours d'acquisition indiquent que l'infertilité serait surtout due aux mâles dont l'arrêt d'activité hivernal serait insuffisant. Leur stockage à $2-3{ }^{\circ} \mathrm{C}$ pendant 2 à 3 mois améliore nettement la fertilité, même avec des femelles à hivernage court. Cependant, il n'y a pas retour aux pontes multiples, même pour des femelles à hivernage plus long.

\section{2 / Elevage des têtards}

Cette phase du cycle ne présente pas de difficulté particulière. Quel que soit le type de bac où les têtards sont élevés, il faut un léger renouvellement de l'eau (50 \% par jour) et éviter les refus alimentaires. L'effet de la densité sur la croissance des têtards est important, pour $1 \mathrm{~m}^{2}$ :

$\mathrm{d} \geq 1000$

poids moyen final : $0,5-0,8 \mathrm{~g}$

$400<\mathrm{d}<600$ poids moyen final : $1,2-1,5 \mathrm{~g}$

$100<\mathrm{d}<200$ poids moyen final : $2,0-2,7 \mathrm{~g}$.

Ces valeurs sont généralement plus faibles que celles observés en étang où les densités sont inférieures et où les poids individuels à la métamorphose peuvent atteindre 6 à $7 \mathrm{~g}$.

Le développement des têtards dure 2 à 3 mois. Une température insuffisante ralentit leur croissance et des mortalités peuvent apparaître au-dessous de $12-15^{\circ} \mathrm{C}$, surtout chez les têtards hivernants ou les jeunes individus au début du printemps. La hauteur d'eau ne semble pas avoir d'influence tant que le renouvellement est suffisant pour l'évacuation des déchets métaboliques.

Les têtards sont microphages, ils ingèrent les algues et bactéries à la surface des supports (parois des bacs, végétaux ...). Ils acceptent parfaitement les granulés à poissons, de préférence non extrudés. Le coefficient de conversion (rapport du poids sec de l'aliment au poids frais des têtards), en élevage sous serre froide, est de 0,9 à 1,2. La distribution de l'aliment doit être régulière, en relation avec la consommation. Tout excès peut en effet provoquer des mortalités, surtout en période de refroidissement, en particulier au début de l'élevage au printemps.

La métamorphose peut débuter fin juin (dans les conditions climatiques de la Bretagne, serre sans chauffage), elle est à son maximum en août/septembre (figure 1).
Figure 1. Taux de métamorphose au cours du temps pour la génération 2000.

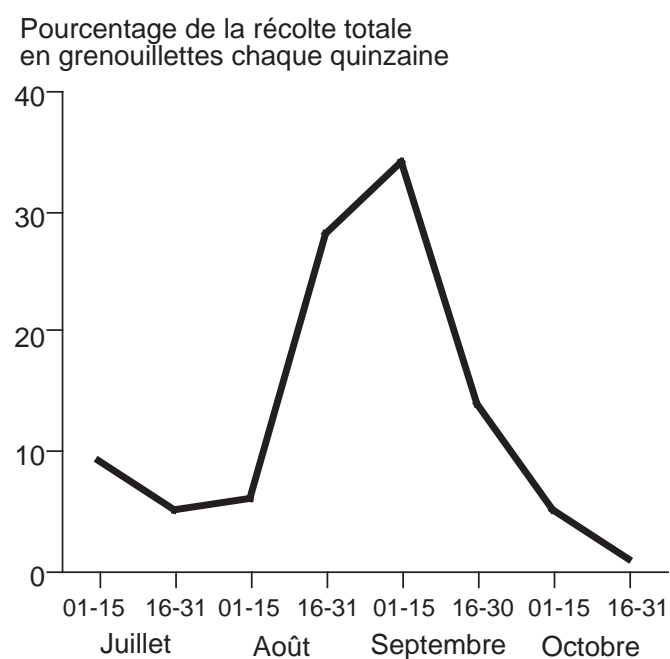

\section{3 / Elevage des grenouillettes}

La récolte s'effectue en continu par des pièges adaptés dans lesquels les grenouillettes peuvent rester deux semaines. Leur transfert dans les bacs de sevrage doit se faire sur la base de l'état de résorption de la queue, car il n'y a pas prise de nourriture tant que celle-ci n'est pas complète. L'homogénéisation des lots à ce stade d'évolution permet d'éviter la création trop rapide d'une hiérarchie aux effets négatifs.

La souche d'élevage accepte, dès la métamorphose, des petits granulés (1 à 1,2 mm), il faut donc des animaux à ouverture buccale suffisante $(>1 \mathrm{~g})$. La densité de stockage des grenouillettes peut être forte : 600 à 1000 individus $/ \mathrm{m}^{2}$. Rapidement, des animaux anorexiques et/ou dominés apparaissent (en 2 à 3 semaines) et représentent de 8 à $10 \%$ des lots de départ, la sélection massale ayant fortement réduit ces chiffres au fil des années (environ $70 \%$ pour les premiers lots sevrés sur granulés). Ces animaux, s'ils sont retirés à temps, peuvent reprendre leur croissance avec une chance de survie de l'ordre de $50 \%$, ce qui laisse supposer qu'ils sont dominés par les autres individus. Pour mesurer le degré de réussite du sevrage, un bilan peut être effectué vers 40 jours après le début de l'alimentation, de façon à comparer éventuellement diverses origines (tableau 4). Sur l'ensemble des 12 croisements, la survie moyenne est de $92 \%$ et le taux d'animaux sevrés aptes à être élevés est en moyenne de $84 \%$ pour une taille moyenne de $32,7 \mathrm{~mm}$ $(\zeta=1,45$ inter-lots, $\zeta=3,44$ en moyenne intralots). Des différences de croissance peuvent ainsi apparaître (cf tableau 4), par exemple entre les lots 1 et 9 (test de Student $t=6,85$; $\mathrm{p}<0,001)$, y compris pour la dispersion des tailles, par exemple le lot 1 est plus hétérogène que le lot $7(\mathrm{~F}=2,64 ; \mathrm{p}<0,005)$.

Le facteur de réussite du sevrage repose beaucoup sur la qualité hygiénique (renouvellement de l'eau, absence de déchets) et sur le calibrage initial des animaux qui permet d'éviter à terme le phénomène de cannibalisme des dominants sur les dominés, avec souvent la mort des deux. 
Tableau 4. Bilan à 40 jours du sevrage de divers lots (REF) de grenouillettes ayant subi des contraintes d'élevage différentes (densité, précocité ...).

$N_{0}=$ nombre initial, $N S=$ nombre de sevrées, $N D=$ nombre de dominées, $N F=$ nombre final, $T=$ longueur moyenne en $\mathrm{mm}, \zeta=$ écart type en $\mathrm{mm}$.

\begin{tabular}{|c|c|c|c|c|c|c|}
\hline REF & $\mathrm{N}_{0}$ & $\mathrm{NS}$ & $\mathrm{ND}$ & $\mathrm{NF}$ & $\mathrm{T}$ & $\zeta$ \\
\hline 1 & 50 & 49 & 1 & 50 & 35,80 & 4,73 \\
\hline 2 & 50 & 43 & 0 & 43 & 34,05 & 3,69 \\
\hline 3 & 50 & 47 & 3 & 50 & 32,02 & 2,99 \\
\hline 4 & 50 & 47 & 3 & 50 & 33,06 & 3,24 \\
\hline 5 & 50 & 43 & 0 & 43 & 31,27 & 3,65 \\
\hline 6 & 50 & 40 & 6 & 46 & 33,18 & 3,18 \\
\hline 7 & 50 & 44 & 3 & 47 & 32,95 & 2,91 \\
\hline 8 & 50 & 42 & 5 & 47 & 33,09 & 3,65 \\
\hline 9 & 50 & 40 & 7 & 47 & 29,91 & 2,95 \\
\hline 10 & 50 & 37 & 10 & 47 & 33,20 & 4,13 \\
\hline 11 & 50 & 42 & 3 & 45 & 32,13 & 3,19 \\
\hline 12 & 50 & 33 & 9 & 42 & 32,03 & 3,33 \\
\hline
\end{tabular}

\section{4 / Elevage des adultes}

Les grenouilles sevrées sont transférées dans des bacs de croissance assez profonds (minimum 50-60 cm) pour éviter les fuites, avec une faible épaisseur d'eau (environ $1 \mathrm{~cm}$ ), un léger renouvellement de l'eau et un nettoyage complet une fois par semaine. Les granulés (30 à $35 \%$ de protéines) sont distribués une fois par jour, leur taille étant adaptée à la croissance ( 2 à $8 \mathrm{~mm}$ ). Dans les conditions actuelles, le taux de conversion est de 1,5 kg de granulés pour produire $1 \mathrm{~kg}$ de pré-adultes.

Au cours des premiers mois d'élevage intensif, la croissance peut être variable selon l'origine des parents et les conditions d'élevage (tableau 5). La comparaison de quelques populations $(\mathrm{G})$ issues de couples sauvages (G1, G2), d'élevage (G4, G5, G6) ou de couples mixtes (G3, G7) montre une forte influence de la sélection sur la récolte finale.
Les produits de couples d'élevage RR (G4, G5, G6) présentent une survie et une croissance supérieures à ceux d'animaux sauvages RR (G1, G2). Cependant, à faible densité, la croissance peut être comparable (G2). Le rétro-croisement d'une femelle d'élevage avec un mâle hybride RLL (G7) (triploïde pour la fertilité), donne une faible survie et deux types d'animaux : RR à croissance semblable à celle des individus d'élevage et RLL à croissance très faible. En revanche, l'hybridation entre des RR sauvages et d'élevage (G3) donne des résultats satisfaisants. Les récoltes élevées obtenues de G3 à G6 ne préjugent pas de la structure démographique, puisque G3 ne présente que $8 \%$ d'individus commercialisables (taille de $30 \mathrm{~g}$ ).

\section{4 / L'élevage de $R$. temporaria}

La grenouille rousse présente des caractéristiques intéressantes :

- ponte dans l'eau puis vie terrestre (prairie, bois),

- homing plus précis que celui des saumons, - l'espèce est bien définie (sans hybride).

Aussi depuis de nombreuses années, cette espèce est élevée dans des conditions de ranching, c'est-à-dire très peu interventionnistes. Il suffit de posséder un étang vidangeable, de le mettre en eau pour la reproduction, de protéger au mieux les têtards de la prédation, de distribuer une alimentation complémentaire, de laisser le flux de grenouillettes partir à la conquête des espaces terrestres favorables et de vider l'étang (mai-juin). Après 2 à 3 ans, les géniteurs reviennent pondre dans leur étang de naissance remis en eau et peuvent être capturés après la ponte. Ce système est autorisé sous certaines conditions de l'arrêté du 5 juin 1985 .

Des essais reposant sur ces principes sont effectués à Rennes depuis 1986 de façon à comprendre le fonctionnement du système.

\section{1 / La reproduction}

La zone expérimentale est constituée d'un lambeau forestier de 23 ha, isolé au milieu

Tableau 5. Croissance des adultes et survie selon l'origine des couples ( $S=$ Sauvage, $E=$ Elevage) et la densité initiale en grenouillettes sevrées (bacs circulaires de $0,64 \mathrm{~m}^{2}$, température $=18^{\circ}$ à $20^{\circ} \mathrm{C}$ ).

$N_{0}=$ nombre initial, $P=$ poids moyen individuel, $B T=$ biomasse récoltée, $H 1=$ fréquence des animaux $\geq$ $60 \mathrm{~mm}= \pm 30 \mathrm{~g}, \mathrm{H} 2$ = fréquence des animaux $\geq 70 \mathrm{~mm}= \pm 50 \mathrm{~g}$.

\begin{tabular}{|c|c|c|c|c|c|c|c|c|c|c|c|}
\hline \multirow[b]{2}{*}{ Lot } & \multicolumn{2}{|c|}{ Octobre } & \multicolumn{2}{|c|}{ Février } & \multicolumn{2}{|c|}{ Juillet } & \multicolumn{5}{|c|}{ Octobre (récolte) } \\
\hline & Couple & $\begin{array}{l}\mathbf{N}_{\mathbf{0}} \\
/ \mathrm{m}^{2}\end{array}$ & $\begin{array}{c}\text { Survie } \\
(\%)\end{array}$ & $\begin{array}{l}\mathbf{P} \\
(\mathrm{g})\end{array}$ & $\begin{array}{c}\text { Survie } \\
(\%)\end{array}$ & $\begin{array}{l}\mathbf{P} \\
(\mathrm{g})\end{array}$ & $\begin{array}{c}\text { Survie } \\
(\%)\end{array}$ & $\begin{array}{l}\mathbf{P} \\
(\mathrm{g})\end{array}$ & $\begin{array}{c}\text { BT } \\
\left(\mathrm{kg} / \mathrm{m}^{2}\right)\end{array}$ & $\begin{array}{l}\text { H1 } \\
(\%)\end{array}$ & $\begin{array}{l}\mathrm{H} 2 \\
(\%)\end{array}$ \\
\hline G1 & $S \times S$ & 550 & 65 & 5 & - & 9,9 & 60 & 16,3 & 5,4 & 0 & 0 \\
\hline G2 & $S \times S$ & 780 & 42 & 6,2 & - & 13,3 & 35 & 31,4 & 8,6 & 60 & 0 \\
\hline G3 & $S \times E$ & 1400 & 86 & 7,2 & - & 17,4 & 64 & 25,3 & 23,3 & 8 & 0 \\
\hline G4 & $E \times E$ & 1000 & 98 & 22,9 & - & 34,4 & 81 & 36,6 & 30,2 & 92 & 28 \\
\hline G5 & $E \times E$ & 1000 & 89 & 32,6 & - & 38 & 77 & 43,7 & 31,3 & 98 & 76 \\
\hline G6 & $E \times E$ & 1400 & 76 & 14,7 & - & 29,2 & 57 & 36 & 28,9 & 88 & 39 \\
\hline G7 & $E \times R L L$ & 1000 & 40 & 3,7 & 12,4 & $\begin{array}{l}\text { RLL } 4,1 \\
\text { RR } 22,2\end{array}$ & 11,7 & $\begin{array}{c}7,2 \\
33,9\end{array}$ & $\begin{array}{l}1,2 \\
0,4\end{array}$ & $\begin{array}{c}0 \\
73\end{array}$ & $\begin{array}{l}0 \\
0\end{array}$ \\
\hline
\end{tabular}


d'une zone expérimentale agricole de l'INRA. Le massif est une futaie (hêtre, chêne) avec un taillis âgé (charme, châtaignier ...). Le sol est donc dégagé avec quelques ronces et buissons (houx, rhododendrons ...).

Le milieu aquatique se résume à des ornières, des flaques temporaires (janvieravril) et de petites dépressions où l'eau peut être permanente certaines années.

L'espèce a été introduite en 1986 à partir de grenouillettes d'élevage intensif issues d'animaux locaux (forêt de Rennes). Depuis, les géniteurs viennent pondre à partir de leur $2^{\text {ème }}$ été ; leur taille est faible (environ $60 \mathrm{~mm}$ ) avec une fécondité de 800 à 1200 œufs par femelle, mais avec une forte fertilité (80 à $100 \%$ d'éclosions). Les géniteurs peuvent pondre 2 à 3 années de suite, dès que la température de l'air atteint $8-9^{\circ} \mathrm{C}$ (Le Garff 1998), soit en janvier-février pour la Bretagne. Du reste, sous l'influence du changement climatique, ces pontes sont de plus en plus précoces pour l'ouest de la France : elles ont débuté fin décembre en 2002 et 2003. Les œufs sont ensuite récoltés pour l'élevage intensif des têtards.

\section{2 / Elevage des têtards}

Les têtards doivent être élevés dans une zone ombragée à des températures inférieures à $20-22^{\circ} \mathrm{C}$, dans des bacs de pisciculture avec 40 à $50 \mathrm{~cm}$ d'eau. Au début du développement, ils se nourrissent de la gélatine des oufs, très abondante chez cette espèce, et l'alimentation artificielle ne doit commencer que très progressivement, lorsque la gélatine devient rare. Cette espèce est particulièrement sensible à tous les excès et déséquilibres de l'eau dont le renouvellement doit être permanent ( 2 à 3 volumes/jour). Les densités d'élevage peuvent être élevées $\left(10000 / \mathrm{m}^{2}\right)$ mais les grenouillettes produites sont vite trop petites $(0,2$ à $0,3 \mathrm{~g})$. La densité optimale semble être de 2000 à $3000 / \mathrm{m}^{2}$, de façon à obtenir des grenouillettes de 0,4 à $0,5 \mathrm{~g}$. La survie de l'œuf à la métamorphose est élevée, autour de $70 \%$ en moyenne. La période de métamorphose est restreinte (2 à 3 semaines en avril-mai), elle ne s'étale pas pour compenser l'effet de la densité qui influe plus sur la taille finale de $R$. temporaria, contrairement aux grenouilles vertes.

Les grenouillettes sont très fragiles, sensibles au soleil, aussi les lâchers s'effectuent dès le stade 4 pattes, lorsque la respiration devient aérienne. Les animaux sont alors déposés dans les points d'eau, début mai, sous ombrage local pour réduire leur visibilité par les oiseaux prédateurs (merles, étourneaux ...).

\section{3/ La vie adulte : taux de retour}

Les informations récoltées portent d'une part sur le nombre de grenouillettes relâchées, la date du lâcher, sur le nombre de pontes déposées au retour des adultes (qui permet de déterminer le nombre de femelles matures), les dates de pontes, mais aussi sur l'estimation (par marquage) du nombre de mâles sur les divers sites. Par ailleurs, les adultes présents sont mesurés, quelques prélèvements de doigts permettent d'estimer les âges, afin d'analyser la structure de la population. Quelques couples sont éventuellement transportés au laboratoire pour étudier la fécondité en rapport avec la taille des géniteurs et suivre le taux d'éclosion de pontes bien individualisées.

Les principales informations recueillies au fil des années (de 1986 à 2004) montrent :

- une croissance faible des adultes dont les tailles restent entre 55 et $72 \mathrm{~mm}$ pour des âges de 2 à 5 ans ;

- une très grande fidélité au site de lâcher. Ainsi une expérience ponctuelle, avec un demi bidon de 200 litres, enterré et rempli d'eau, dans lequel 1000 grenouillettes ont été introduites, a permis le retour d'une dizaine de couples après 2 étés. Ce test a été effectué dans une zone sèche, sans eaux même temporaires, donc a priori inapte à la reproduction de l'espèce ;

- un faible brassage inter-sites au-delà de 50 à 100 m (l'arrêt des lâchers sur un site entraîne un déclin rapide des pontes jusqu'à l'épuisement des derniers géniteurs, soit 2 à 3 ans) ;

- un taux de retour de 2 à $12 \%$, avec une moyenne décennale de $6 \%$.

Globalement, dans une population naturelle stable, la survie de l'œuf à l'adulte est de 2 à $3 \%$, dans le cas présent elle est de $4 \%$ en moyenne compte tenu de l'amélioration de la survie des têtards.

A partir d'une expérience en grandeur réelle en Bourgogne (2002, Martial comm. pers.), il est possible de comparer les résultats :

\begin{tabular}{|l|c|c|}
\hline & Bourgogne & Rennes \\
\hline $\mathrm{Nb}$ femelles & 5 & 4 \\
$\mathrm{Nb}$ œufs $/ \mathrm{m}^{2}$ & 10000 & 4000 \\
$\mathrm{Nb}$ grenouillettes & $\begin{array}{c}\text { (bassins en terre) } \\
1000 \\
(1 \mathrm{~kg} \text { aliment })\end{array}$ & $\begin{array}{c}3000 \\
(4 \mathrm{~kg} \text { aliment })\end{array}$ \\
\hline $\begin{array}{l}\text { Nature } \\
\mathrm{Nb} \text { géniteurs 2+ }\end{array}$ & $40(1 \mathrm{~kg})$ & $180(4,5 \mathrm{~kg})$ \\
\hline
\end{tabular}

Ces chiffres ne sont qu'indicatifs sachant que bien des paramètres restent à vérifier dans les conditions normales du ranching, en particulier les taux de survie, estimés ici par le pisciculteur. Par ailleurs, l'exploitation régulière de la population exerce une pression sélective avec rajeunissement des reproducteurs et baisse de la fécondité individuelle (Augert 1992).

\section{Conclusions générales : les contraintes d'un élevage}

Face à la réduction des stocks naturels, l'élevage pourrait être un recours pour protéger les populations naturelles surexploitées. Il pourrait aussi servir à des reconstructions d'effectifs (Neveu, sous presse).

A ce jour les conditions sont favorables au développement de cette activité du fait de la 
chute de production dans les élevages en Chine (due à des pathologies), de la réduction des stocks péri-méditerranéens et de l'augmentation des prix des grenouilles vivantes.

Les objectifs pourraient donc être de : - développer une filière traditionnelle «cuisses fraîches»,

- freiner la diminution des stocks naturels,

- satisfaire la demande (récente) pour l'enseignement,

- reconstituer des stocks sauvages pour la pêche de loisir,

- appliquer les acquis techniques pour des opérations de soutien d'effectifs d'espèces rares, voire de réintroduction de certaines.

Quel élevage choisir?

- soit l'intensif avec ridibunda, mais actuellement la réglementation n'encourage pas ce système puisque les grenouilles vertes (sauf esculenta) sont protégées (arrêté du 22/07/1993) et les dérogations complexes à obtenir, en liaison avec très souvent une méconnaissance totale de la biologie de ces animaux.

Comme toute installation d'élevage intensif, les structures sont d'un coût élevé et leur conception reste à améliorer. Il faut aussi considérer les problèmes de nuisances (chant des géniteurs) et traiter les rejets, sachant que les débits d'eau actuels de l'élevage expérimental sont faibles $\left(15-20 \mathrm{~m}^{3} / \mathrm{t} /\right.$ jour, des essais en cours montrent une réduction possible à 3$5 \mathrm{~m}^{3}$ avec une bonne hygiène).

Les risques pathologiques sont mal connus, même s'il n'existe pas de maladies spécifiques en France, mais des germes ubiquistes (Aeromonas) peuvent donner de fortes mortalités (Ippen et Zwart 1996). Le syndrome dit des pattes rouges apparaît dans des conditions générales de mauvaise hygiène, de malnutrition et, plus généralement, sous l'influence de stress divers. Cependant, l'étiologie semble plus complexe. La mise en évidence de diverses souches et leur permanence sur des animaux bien portants ne permettent pas encore une prophylaxie et un traitement efficaces (Pearson et al 2000).

A priori les coûts de production seront élevés. Cependant des améliorations peuvent être effectuées sur les économies d'énergie, l'alimentation, la reproduction contrôlée, la sélection de souches performantes, etc, c'està-dire toutes les caractéristiques d'autres élevages aquacoles plus développés.

- soit l'extensif avec $R$. temporaria. Pour cette espèce, les obstacle réglementaires sont moins stricts, des autorisations sont prévues à partir de l'arrêté de 1985, qui ne concerne paradoxalement que cette espèce. Des élevages existent déjà (Jura, Dombes, Bourgogne), mais ils dépendent de la pérennité des autorisations. Celles-ci, données pour 3 ans, sont souvent difficiles à renouveler. Les arguments avancés concernent les risques de pollution génétique, alors que les géniteurs sont régionaux, ou encore une dérive génétique : il reste à la mesurer sachant que la pression de sélection reste la même au niveau de la vie adulte. Les coûts de production sont réduits en dehors de la construction des étangs pour les têtards (surface : 1000 à $2000 \mathrm{~m}^{2}$ ). La main-d'œuvre est réduite à la surveillance, à l'alimentation des têtards (3 à 4 mois de développement) et à la récolte des adultes (2 à 3 semaines).

La production est labellisable en «Durable» et «Biologique». Ce type d'élevage est possible à travers toute la France forestière et prairiale avec très peu de surface en eau nécessaire (intérêt du homing et de la forte densité des têtards).

Il reste une petite défiance sur la couleur de la chair (un peu jaunâtre) selon les régions, mais appréciée localement (Lyon). La défiance est plus grande de la part des groupes «protectionnistes» vis-à-vis de cet animal «sauvage» et, par définition, «intouchable», ce qui limite actuellement fortement le développement de cette production de l'aquaculture biologique.

\section{Références}

Amiche M., Delfou A., Nicolas P., 1996. Les trésors de la peau des grenouilles. La Recherche, 291, 72-77.

Augert D., 1992. Variations de la structure démographique de populations voisines de grenouilles rousses (Rana temporaria). Thèse Doc. Univ., Lyon, 142 p.

Chen L.C., 1990. Aquaculture in Taïwan. Blackwell Publishing Ltd, Oxford, $273 \mathrm{p}$.

Collins J.P., Storfer A., 2003. Global amphibian declines: sorting the hypotheses. Diversity and Distributions, 9, 89-98.

Green D.M., 1997. Amphibianes in decline. Canadian studies of a global problem. Herpetological Conservation, 1, 338p.

Hardouin J., 1994. Le commerce international des cuisses de grenouilles dans la CEE de 1988 à 1992. Bull. Rech. Agron. Gembloux, 29, 217-245.

Ippen R., Zwart P., 1996. Infectious and parasitic diseases of captive Reptiles and Amphibians. Rev. Sci. Tech. Off. Int. Epiz., 15, 43-54.

Le Garff B., 1998. Relations entre les facteurs météorologiques et la ponte chez la grenouille rousse Rana temporaria (Amphibiens, Anoures, Ranidés) dans l'ouest de la France (Forêt de Rennes, 35). Bull. Soc. Zool. Fr., 123, 61-71.

Lima S.L., Agostino C.A., 1992. A technologia de Criaçaô de Ràs.Univ. Féd. Vicoça, Brasil, 168 p.
Miaud C., Guyétant R., 1998. Plasticité et sélection sur les traits de vie d'un organisme à cycle vital complexe, la grenouille rousse Rana temporaria (Amphibien : Anoure) Bull. Soc. Zool. Fr., 123, 325-344.

Neveu A., 1997. Lintroduction d'espèces allochtones de grenouilles vertes en France, deux problèmes différents celui de $R$. catesbeiana et celui des taxons non présents du complexe esculenta. Bull. Fr. Pêche Piscic., 344/345, 165-171.

Neveu A., sous presse. Edible Frogs. In : Safran P. (ed), Fisheries and aquaculture: towards Sustainable Aquatic Living Resources Management. Encyclopedia of Life Support Systems (EOLSS), developed under the auspices of the UNESCO. Eolss Publishers, Oxford ,UK

Pearson M.D., Hirono I., Aoki T., Miranda R., Inglis V. 2000. Virulence properties of mobile aeromonads isolated from farmed frogs Rana tigerina and R. rugulosa. Dis. Aquat. Org., 40, 185-193.

Somsueb P., Boonyaratpalin M., 2001. Optimum protein and energy levels for the Thai native frog, $R$. rugulosa Weigmann. Aqua. Res., 32, 33-38.

Viosca P., 1934. Principles of Bullfrog Culture. Southern Biol. Supply, New Orleans, 31 p.

Wasserman F., 1990. La grenouille dans tous ses états. Collection Découvertes Gallimard $n^{\circ} 92$, Editions Gallimard, Paris, 112 p. 


\section{Abstract}

Raniculture or field harvesting? State of the art in France.

In Europe, frogs appeared in the best culinary circles only around the $16^{\text {th }}$ century. During the $20^{\text {th }}$, a strong decline is observed in certain populations under direct and indirect influences of human activities.

France is the main importer with $3000-4000$ tons.year-1 of deep frozen legs from Far East countries and 700-800 tons.year ${ }^{-1}$ of live frogs from the Near East, that are transformed into fresh legs to gastronomic restaurants.

During recent years some countries are carrying out trials of intensive rearing with neotropical species and a recent artificial feeding. In West part of France (near Rennes town) trials began during 1980's with local water frogs (esculenta complex). This complex is composed by two parental species (Rana lessonae, $R$. ridibunda) and a hybridogenetic form ( $R$. esculenta). Pure ridibunda stocks are the most attractive issue for intensive rearing.

At the beginning of our trial, the females gave several clutches within a year with a high fertility. However, after a decade of rearing, fecundity and fertility beca- me reduced, the causes of this problem being not completely identified. The juveniles are fed on pellets. After 40 days, survival rate of froglets is $92 \%$, and their mean size reaches $32 \mathrm{~mm}$. In a green house the growing period from weaned froglets to mature adults is around 10-12 months with a production of 20. 30 kg.m ${ }^{-2}$ (thermic level between 15 and $25{ }^{\circ} \mathrm{C}$ ). Any combination with lessonae genome strongly reduces the final size.

An alternative to intensive production would be to restore natural stocks in suitable areas. Since 1986, several trials with $R$. temporaria have been carried out, which associate intensive rearing of tadpoles in tanks, releasing of metamorphs at a natural site (a small forest area) and control of the return of adults (return rate : $\pm 6 \%$ ) to spawning sites after two summers (strong homing effect).

Discussions are conducted on the best strategy to take up in relation to laws and economic restraints in France.

NEVEU A., 2004. La raniculture est-elle une alternative à la récolte ? Etat actuel en France. INRA Prod. Anim., 17, 167-175. 
